\title{
Lipschitz-Type and Bloch-Type Spaces of Pluriharmonic Mappings in a Hilbert Space
}

\author{
Yong Liu \\ Department of Mathematics, Shaoxing College of Arts and Sciences, Shaoxing 312000, Zhejiang Province, China \\ Correspondence should be addressed to Yong Liu; liuyongsdu1982@163.com
}

Received 12 May 2017; Accepted 11 July 2017; Published 7 August 2017

Academic Editor: Ruhan Zhao

Copyright (C) 2017 Yong Liu. This is an open access article distributed under the Creative Commons Attribution License, which permits unrestricted use, distribution, and reproduction in any medium, provided the original work is properly cited.

We investigate some properties of pluriharmonic mappings in an infinite dimensional complex Hilbert space. Several characterizations for pluriharmonic mappings to be in Lipschitz-type and Bloch-type spaces are given, which are generalizations of the corresponding known ones for holomorphic functions with several complex variables.

\section{Introduction}

Let $E$ be a complex Hilbert space of infinite dimension. Given a subdomain $\Omega$ of $E$, a function $h: \Omega \rightarrow \mathbb{C}$ is said to be holomorphic if it is Fréchet differentiable at each point $z \in \Omega$ or, equivalently, if $h(z)=\sum_{n=1}^{\infty} P_{n}(z)$ for all $z \in \Omega$, where $P_{n}$ is an $n$-homogeneous polynomial.

A continuous complex-valued function $f$ defined on $\Omega$ is said to be pluriharmonic if there are two holomorphic functions $h$ and $g$ on $\Omega$ such that $f=h+\bar{g}$. We denote the class of all pluriharmonic mappings on $\Omega$ by $\mathscr{P}(\Omega)$. Suppose that $\left(e_{k}\right)_{k \in \Gamma}$ is an orthonormal basis of $E$. Then every $z \in E$ can be written as $z=\sum_{k \in \Gamma} z_{k} e_{k}$ and $\bar{z}=\sum_{k \in \Gamma} \overline{z_{k}} e_{k}$. For a pluriharmonic mapping $f \in \mathscr{P}(\Omega)$, we introduce the notion

$$
\begin{aligned}
& \nabla f=\left(\frac{\partial f}{\partial z_{1}}, \ldots, \frac{\partial f}{\partial z_{k}}, \ldots,\right)_{k \in \Gamma}, \\
& \bar{\nabla} f=\left(\frac{\partial f}{\partial \overline{z_{1}}}, \ldots, \frac{\partial f}{\partial \overline{z_{k}}}, \ldots,\right)_{k \in \Gamma} .
\end{aligned}
$$

Let $\omega:[0,+\infty) \rightarrow[0,+\infty)$ be a continuous increasing function with $\omega(0)=0$. We say that $\omega$ is a majorant if $\omega(t) / t$ is nonincreasing for $t>0$. A function $f: \Omega \rightarrow \mathbb{C}$ is said to belong to Lipschitz space $\Lambda_{\omega}(\Omega)$ if there is a positive constant $C$ such that

$$
|f(z)-f(w)| \leq C \omega(|z-w|)
$$

for all $z, w \in \Omega$ (cf. [1]).
Given a proper subdomain $G$ of $E$ and a majorant $\omega$, we say that $G$ is $\omega$-extension if for each pair of points $z, w \in G$ can be joined by a rectifiable curve $\gamma \subset G$ satisfying

$$
\int_{\gamma} \frac{\omega\left(d_{G}(z)\right)}{d_{G}(z)} d s(z)<C \omega(|z-w|)
$$

with some fixed positive constant $C=C(G, \omega)$, where $d s$ stands for the arc length measure on $\gamma$ and $d_{G}(z)$ denotes the distance from $z$ to the boundary $\partial G$ of $G$ (cf. [2]).

In [1], Dyakonov characterized the holomorphic functions in $\Lambda_{\omega}(\mathbb{D})$ in terms of their modulus. Later, Pavlović [3] came up with a relatively simple proof of the results of Dyakonov. For the generalizations of this topic, we refer to [4-6]. In this paper, we consider the corresponding problem in the case of $\mathscr{P}(\Omega)$. Our first result is the following theorem which can be viewed as an extension of [6, Theorem 1] to the infinite dimensional setting.

Theorem 1. Let $\omega$ be a majorant and $\Omega$ be a simply connected $\omega$-extension subdomain of $E$. If $f=h+\bar{g} \in \mathscr{P}(\Omega)$, then the following statements are equivalent:
(a) $f \in \Lambda_{\omega}(\Omega)$.
(b) $h \in \Lambda_{\omega}(\Omega)$ and $g \in \Lambda_{\omega}(\Omega)$.
(c) $|h| \in \Lambda_{\omega}(\Omega)$ and $|g| \in \Lambda_{\omega}(\Omega)$.
(d) $|h| \in \Lambda_{\omega}(\Omega, \partial \Omega)$ and $|g| \in \Lambda_{\omega}(\Omega, \partial \Omega)$. 
Here $\Lambda_{\omega}(G, \partial G)$ denotes the class of continuous functions on $G \cup \partial G$ which satisfy (2) with some positive constant $C$, whenever $z \in G$ and $w \in \partial G$.

Let $\mathbb{B}_{E}$ be the unit ball of $E$. For each $f \in \mathscr{P}\left(\mathbb{B}_{E}\right)$, we denote

$$
\Lambda_{f}(z)=\max _{\theta \in \partial \mathbb{B}_{E}}|\nabla f(z) \cdot \theta+\bar{\nabla} f(z) \cdot \bar{\theta}|
$$

Following [4], the $\omega$ - $\alpha$-Bloch space $\mathscr{B}_{\omega}^{\alpha}$ of $\mathscr{P}\left(\mathbb{B}_{E}\right)$ consists of all functions $f \in \mathscr{P}\left(\mathbb{B}_{E}\right)$ such that

$$
\|f\|_{\omega, \alpha}=\sup _{z \in \mathbb{B}_{E}} \omega\left(\left(1-|z|^{2}\right)^{\alpha}\right) \Lambda_{f}(z)<\infty
$$

and the little $\omega$ - $\alpha$-Bloch space $\mathscr{B}_{\omega, 0}^{\alpha}$ consists of the functions $f \in \mathscr{P}\left(\mathbb{B}_{E}\right)$ such that

$$
\lim _{|z| \rightarrow 1^{-}} \sup _{z \in \mathbb{B}_{E}} \omega\left(\left(1-|z|^{2}\right)^{\alpha}\right) \Lambda_{f}(z)=0 .
$$

In particular, when $f$ is holomorphic and $\omega(t)=t$, the space $\mathscr{B}_{\omega}^{\alpha}$ is $\mathscr{B}^{\alpha}$ of $H\left(\mathbb{B}_{E}\right)$ which has been studied in [7].

Let $h: \mathbb{B}_{E} \rightarrow \mathbb{C}$ be continuous. If there exists a constant $C>0$ such that

$$
\left(1-|z|^{2}\right)^{1 / 2}\left(1-|w|^{2}\right)^{1 / 2} \frac{|h(z)-h(w)|}{|z-w|} \leq C,
$$

for any $z, w \in \mathbb{B}_{E}, z \neq w$, then we say that $h$ satisfies weighted Lipschitz condition (cf. [8]).

In the theory of function spaces, the relationship between Bloch spaces and weighted Lipschitz functions has attracted much attention. In 1986, Holland and Walsh established a standard criterion for analytic Bloch space in the unit disc $\mathbb{D}$ in terms of weighted Lipschitz functions. Since then, a series of work has been carried out to characterize Bloch, $\alpha$ Bloch, little $\alpha$-Bloch, and Besov spaces of holomorphic and harmonic functions along this line. For instance, Ren and Tu [9] extended Holland and Walsh's criterion to the Bloch space in the unit ball of $\mathbb{C}^{n}, \mathrm{Li}$ and Wulan [10] and Zhao [11] characterized holomorphic $\alpha$-Bloch space in terms of $\left(1-|z|^{2}\right)^{\beta}\left(1-|w|^{2}\right)^{\alpha-\beta}|f(z)-f(w)| /|z-w|$. For the related results of harmonic functions, we refer to $[4,12-14]$ and the references therein.

The second purpose of this paper is to consider the corresponding problems for pluriharmonic mappings in an infinite dimensional complex Hilbert space E. In Section 2, we collect some known results that will be needed in the sequel. Our main results and their proofs are presented in Sections 3 and 4.

Throughout this paper, constants are denoted by $C$, and they are positive and may differ from one occurrence to the other. The notation $A \asymp B$ means that there exists a positive constant $C$ such that $B / C \leq A \leq C B$.

\section{Preliminaries}

We need the following preliminary material (see $[7,8]$ for the details).
For $a \in \mathbb{B}_{E}$, the involution $\varphi_{a}: \mathbb{B}_{E} \rightarrow \mathbb{B}_{E}$ is defined as

$$
\varphi_{a}(z)=\left(s_{a} Q_{a}+P_{a}\right)\left(m_{a}(z)\right),
$$

where $s_{a}=\sqrt{1-|a|^{2}}$ and $m_{a}: \mathbb{B}_{E} \rightarrow \mathbb{B}_{E}$ is the analytic map

$$
m_{a}(z)=\frac{a-z}{1-\langle z, a\rangle},
$$

$P_{a}: E \rightarrow E$ is the orthogonal projection along the onedimensional subspace spanned by $a$, more precisely,

$$
P_{a}(z)=\frac{\langle z, a\rangle}{\langle a, a\rangle} a
$$

and $Q_{a}$ is the orthogonal complement, and $Q_{a}=I d-P_{a}$. The automorphisms of the unit ball $\mathbb{B}_{E}$ turn to be compositions of such analogous involutions with unitary transformations of $E$.

As in the finite dimensional case, the pseudohyperbolic and hyperbolic metrics on $\mathbb{B}_{E}$ are, respectively, defined by

$$
\begin{aligned}
& \rho_{E}(z, w)=\left|\varphi_{z}(w)\right|=\left|\varphi_{w}(z)\right|, \\
& \beta_{E}(z, w)=\frac{1}{2} \ln \frac{1+\rho_{E}(z, w)}{1-\rho_{E}(z, w)},
\end{aligned}
$$

$$
z, w \in \mathbb{B}_{E}
$$

It is known (see [7]) that

$$
\begin{aligned}
& \left|\varphi_{z}(w)\right|^{2}=1-\frac{\left(1-|z|^{2}\right)\left(1-|w|^{2}\right)}{|1-\langle z, w\rangle|^{2}} \\
& \quad=\frac{-2 \Re\langle z, w\rangle+|\langle z, w\rangle|^{2}+|z|^{2}+|w|^{2}-|z|^{2}|w|^{2}}{|1-\langle z, w\rangle|^{2}} \\
& \quad \leq \frac{|z-w|^{2}}{|1-\langle z, w\rangle|^{2}} .
\end{aligned}
$$

For each $a \in \mathbb{B}_{E}$ and $r \in(0,1)$, we define the pseudohyperbolic ball with center $a$ and radius $r$ as

$$
E(a, r)=\left\{z \in \mathbb{B}_{E}: \rho_{E}(a, z)<r\right\} .
$$

A simple computation gives that $E(a, r)$ is a Euclidean ball with center and radius given by

$$
\begin{aligned}
& \frac{1-r^{2}}{1-r^{2}|z|^{2}} z \\
& \frac{1-|z|^{2}}{1-r^{2}|z|^{2}} r .
\end{aligned}
$$

The following lemma will be needed in the sequel. See [15] for the analogue of this result in several complex variables.

Lemma 2. Let $r \in(0,1)$ and $w \in E(z, r)$. Then $1-|z|^{2}=$ $1-|w|^{2} \asymp|1-\langle z, w\rangle|$. 
Proof. From (15), we have $\left|\varphi_{z}(w)\right|<r$. It follows from (12) that

$$
\begin{aligned}
\frac{1-r^{2}}{4} & <\frac{\left(1-|z|^{2}\right)\left(1-|w|^{2}\right)}{4|1-\langle z, w\rangle|^{2}} \\
& \leq \frac{\left(1-|z|^{2}\right)\left(1-|w|^{2}\right)}{4(1-|z|)^{2}} \leq \frac{\left(1-|w|^{2}\right)}{\left(1-|z|^{2}\right)} .
\end{aligned}
$$

Similarly, we can obtain that $\left(1-r^{2}\right) / 4<\left(1-|z|^{2}\right) /\left(1-|w|^{2}\right)$. Combining these two inequalities with (12), we have

$$
1-|z|^{2}=1-|w|^{2}=|1-\langle z, w\rangle| .
$$

The following lemma comes from [4].

Lemma 3. Let $\omega(t)$ be a majorant and $u \in(0,1]$ and $v \in$ $(1, \infty)$. Then, for $t \in(0, \infty)$,

$$
\begin{aligned}
& \omega(u t) \geq u \omega(t), \\
& \omega(v t) \leq v \omega(t) .
\end{aligned}
$$

A combination of Lemmas 2 and 3 yields the following.

Lemma 4. Let $r \in(0,1)$ and $u, v \in E(z, r)$. Then $\omega\left(1-|u|^{2}\right)=$ $\omega\left(1-|v|^{2}\right)$.

\section{Lipschitz Spaces}

We begin this section with some lemmas which will be used in the proof of Theorem 1.

\subsection{Several Lemmas}

Lemma 5. Let $f$ be a real pluriharmonic function of $\mathbb{B}_{E}$ with $|f|<1$. Then, for each $\theta \in \partial \mathbb{B}_{E}$,

$$
|\nabla f(z) \cdot \theta| \leq \frac{4}{\pi} \frac{1-|f(z)|^{2}}{1-|z|^{2}} .
$$

Proof. For a fixed $\theta \in \partial \mathbb{B}_{E}$, let $F(\zeta)=f(\theta \zeta)$ in the unit disc $\mathbb{D}$. Then $F$ is a harmonic function on $\mathbb{D}$ with $|F(\zeta)|<1$. It follows from [16] that

$$
\left|F_{\zeta}\right| \leq \frac{4}{\pi} \frac{1-|F(\zeta)|^{2}}{1-|\zeta|^{2}},
$$

which implies that

$$
|\nabla f(z) \cdot \theta| \leq \frac{4}{\pi} \frac{1-|f(z)|^{2}}{1-|z|^{2}},
$$

where $z=\theta \zeta$. This completes the proof.

Lemma 6. Let $\omega$ be a majorant and $\Omega$ be a w-extension domain of $E$. If $g=u+i v$ be a holomorphic function on $\Omega$ and $u \in \Lambda_{\omega}(\Omega)$, then $g \in \Lambda_{\omega}(\Omega)$.
Proof. Fixing a point $z \in \Omega$ and considering the function $U$, defined on $\mathbb{B}_{E}$ by

$$
U(w)=\frac{u(z+d(z) w)}{M_{z}}, \quad w \in \mathbb{B}_{E},
$$

here

$$
\begin{aligned}
d(z) & =d_{\Omega}(z), \\
M_{z} & =\sup \{|u(\zeta)|:|\zeta-z|<d(z)\} .
\end{aligned}
$$

Since $U$ is pluriharmonic in $\mathbb{B}_{E}$ and $|U(w)|<1$, by Lemma 5, we have that, for each $\theta \in \partial B_{E}$,

$$
|\nabla U(0) \cdot \theta| \leq \frac{4}{\pi}\left(1-|U(0)|^{2}\right) \leq \frac{8}{\pi}(1-|U(0)|),
$$

which in turn gives

$$
d(z)|\nabla u(z) \cdot \theta| \leq \frac{8}{\pi}\left(M_{z}-|u(z)|\right) .
$$

Hence

$$
d(z)|\nabla g(z) \cdot \theta| \leq \frac{16}{\pi}\left(M_{z}-|u(z)|\right) .
$$

By the assumption $u \in \Lambda_{\omega}(\Omega)$, we have, for each $\zeta \epsilon$ $\mathbb{B}(z, d(z))$,

$$
|u(\zeta)|-|u(z)| \leq|u(\zeta)-u(z)| \leq C \omega(d(z)),
$$

which implies that

$$
M_{z}-|u(z)| \leq C \omega(d(z)) .
$$

Thus, for any $\theta \in \partial B_{E}$, we have

$$
d(z)|\nabla g(z) \cdot \theta| \leq C \omega(d(z)), \quad z \in \Omega .
$$

For a pair of points $z_{1}, z_{2} \in \Omega$, we let $\gamma$ be a rectifiable curve which joins $z_{1}$ and $z_{2}$ satisfying (2). Integrating (30) along $\gamma$ leads to

$$
\left|g\left(z_{1}\right)-g\left(z_{2}\right)\right| \leq C \int_{\gamma} \frac{\omega(d(z))}{d(z)} d s(z) .
$$

Combining (31) with (3), we have

$$
\left|g\left(z_{1}\right)-g\left(z_{2}\right)\right| \leq C \omega\left(\left|z_{1}-z_{2}\right|\right),
$$

which completes the proof.

As an application of Lemma 6, we can obtain the following.

Lemma 7. Let $\omega$ be a majorant and let $f=h+\bar{g}$ be a pluriharmonic mapping on a simply connected $\omega$-extension domain $\Omega$. Then $f \in \Lambda_{\omega}(\Omega)$ if and only if both $g, h \in \Lambda_{\omega}(\Omega)$. 
Proof. We only need to prove necessity since the sufficiency is obvious. Let $f=h+\bar{g}=u+i v$, where $u, v$ are real. As $f, \bar{f} \in \Lambda_{\omega}(\Omega)$ and

$$
\begin{aligned}
& u=\frac{f+\bar{f}}{2}=\frac{h+g+\overline{h+g}}{2}, \\
& v=\frac{f-\bar{f}}{2 i}=\frac{(h-g)-\overline{(h-g)}}{2 i},
\end{aligned}
$$

by Lemma 6 , we see that $g, h \in \Lambda_{\omega}(\Omega)$.

The following lemma is an analogue of [5, Theorem 1] for holomorphic functions in an infinite dimensional Hilbert space $E$. Since the proof is almost the same as the one in [5], we leave it to the readers.

Lemma 8. Let $\omega$ be a majorant and $\Omega$ be a simply connected $\omega$-extension domain of $E$. If $h$ is a holomorphic function on $\Omega$, then the following statements are equivalent:

(i) $h \in \Lambda_{\omega}(\Omega)$.

(ii) $|h| \in \Lambda_{\omega}(\Omega)$.

(iii) $|h| \in \Lambda_{\omega}(\Omega, \partial \Omega)$,

where $\Lambda_{\omega}(\Omega, \partial \Omega)$ denotes the class of continuous functions on $\Omega \cup \partial \Omega$ which satisfy (2) with some positive constant $C$, whenever $z \in \Omega$ and $w \in \partial \Omega$.

\subsection{The Proof of Theorem 1}

Proof. The proof follows from Lemmas 5-8.

\section{Bloch Spaces}

In this section, we show some characterizations of the spaces $\mathscr{B}_{\omega}^{\alpha}$ and $\mathscr{B}_{\omega, 0}^{\alpha}$ in terms of $|f(z)-f(w)| /|z-w|$ on the unit ball of $\mathbb{B}_{E}$. We first extend [4, Theorem 3] to the setting of $\mathscr{P}\left(\mathbb{B}_{E}\right)$ as follows.

Theorem 9. Let $f \in \mathscr{P}\left(\mathbb{B}_{E}\right), 0 \leq \beta<1$, and $\beta \leq \alpha<1+\beta$. Then $f \in \mathscr{B}_{\omega}^{\alpha}$ if and only if

$$
\begin{aligned}
L= & \sup _{z, w \in \mathbb{B}_{E}, z \neq w} \omega\left(\left(1-|z|^{2}\right)^{\beta}\left(1-|w|^{2}\right)^{\alpha-\beta}\right) \\
& \cdot \frac{|f(z)-f(w)|}{|z-w|}<\infty .
\end{aligned}
$$

Proof. For a fixed point $z$ in (34), we can find $w \in \mathbb{B}_{E}$ which satisfies that $w=z+r \theta_{0}$ and

$$
\Lambda_{f}(z)=\left|\nabla f(z) \cdot \theta_{0}+\bar{\nabla} f(z) \cdot \overline{\theta_{0}}\right|,
$$

where $r>0$, and $\theta_{0} \in \partial \mathbb{B}_{E}$. Consequently, we have

$$
\begin{aligned}
\omega( & \left.\left(1-|z|^{2}\right)^{\beta}\left(1-|w|^{2}\right)^{\alpha-\beta}\right)|f(z)-f(w)| \\
& =\omega\left(\left(1-|z|^{2}\right)^{\beta}\left(1-|w|^{2}\right)^{\alpha-\beta}\right) \\
& \cdot\left|\int_{\overline{z w}} f_{\zeta}(\zeta) d \zeta+f_{\bar{\zeta}}(\zeta) d \bar{\zeta}\right| .
\end{aligned}
$$

By letting $r \rightarrow 0$, we deduce that

$$
\omega\left(\left(1-|z|^{2}\right)^{\alpha}\right) \Lambda_{f}(z) \leq L
$$

Conversely, we assume that $f \in \mathscr{B}_{\omega}^{\alpha}$. For $z, w \in \mathbb{B}_{E}$,

$$
\begin{aligned}
& |f(z)-f(w)|=\left|\int_{\overline{z w}} f_{\zeta}(\zeta) d \zeta+f_{\bar{\zeta}}(\zeta) d \bar{\zeta}\right| \\
& \quad \leq|z-w| \int_{0}^{1} \Lambda_{f}(w+t(z-w)) d t \\
& \quad \leq C|z-w|\|f\|_{\omega, \alpha} \int_{0}^{1} \frac{d t}{\omega\left(\left(1-|t z+(1-t) w|^{2}\right)^{\alpha}\right)} .
\end{aligned}
$$

Since, for $z, w \in \mathbb{B}_{E}$ and $t \in[0,1]$,

$$
\begin{aligned}
(1 & \left.-|t z+(1-t) w|^{2}\right)^{\alpha} \geq(1-|t z+(1-t) w|)^{\alpha} \\
& \geq(t(1-|z|)+(1-t)(1-|w|))^{\alpha} \\
& \geq\left(t\left(\frac{1-|z|^{2}}{2}\right)+(1-t)\left(\frac{1-|w|^{2}}{2}\right)\right)^{\alpha} \\
& \geq\left(\frac{t}{2}\right)^{\beta}\left(\frac{1-t}{2}\right)^{\alpha-\beta}\left(1-|z|^{2}\right)^{\beta}\left(1-|w|^{2}\right)^{\alpha-\beta},
\end{aligned}
$$

we get

$$
\begin{aligned}
& \frac{|f(z)-f(w)|}{|z-w|} \leq C \int_{0}^{1} \frac{d \mathrm{t}}{\omega\left(\left(1-|t z+(1-t) w|^{2}\right)^{\alpha}\right)} \\
& \leq C \int_{0}^{1} \frac{d t}{\omega\left((t / 2)^{\beta}((1-t) / 2)^{\alpha-\beta}\left(1-|z|^{2}\right)^{\beta}\left(1-|w|^{2}\right)^{\alpha-\beta}\right)} \\
& \leq \frac{C}{\omega\left(\left(1-|z|^{2}\right)^{\beta}\left(1-|w|^{2}\right)^{\alpha-\beta}\right)} \int_{0}^{1} \frac{d s}{t^{\beta}(1-t)^{\alpha-\beta}} \\
& \leq \frac{C}{\omega\left(\left(1-|z|^{2}\right)^{\beta}\left(1-|w|^{2}\right)^{\alpha-\beta}\right)},
\end{aligned}
$$

where the last integral converges since $\alpha<1+\beta$. Thus

$$
\omega\left(\left(1-|z|^{2}\right)^{\beta}\left(1-|w|^{2}\right)^{\alpha-\beta}\right) \frac{|f(z)-f(w)|}{|z-w|}<\infty .
$$

This completes the proof of Theorem 9.

Theorem 10. Let $f \in \mathscr{P}\left(\mathbb{B}_{E}\right), 0 \leq \beta<1$, and $k u \beta \leq \alpha<1+\beta$. Then $f \in \mathscr{B}_{\omega, 0}^{\alpha}$ if and only if

$$
\begin{gathered}
\lim _{|z| \rightarrow 1^{-}} \sup _{w \in \mathbb{B}_{E}, z \neq w} \omega\left(\left(1-|z|^{2}\right)^{\beta}\left(1-|w|^{2}\right)^{\alpha-\beta}\right) \\
\cdot \frac{|f(z)-f(w)|}{|z-w|}=0 .
\end{gathered}
$$


Proof.

Sufficiency. Assume that (42) holds. Then, for any $\epsilon>0$, there exists $\delta \in(0,1)$ such that

$$
\begin{aligned}
& \sup _{w \in \mathbb{B}_{E}, z \neq w} \omega\left(\left(1-|z|^{2}\right)^{\beta}\left(1-|w|^{2}\right)^{\alpha-\beta}\right) \frac{|f(z)-f(w)|}{|z-w|} \\
& \quad<\epsilon
\end{aligned}
$$

whenever $\delta<|z|<1$. It follows by an argument similar to that in the proof of Theorem 9 that we have

$$
\begin{aligned}
& \omega\left(\left(1-|z|^{2}\right)^{\alpha}\right) \Lambda_{f}(z) \\
& <C \sup _{w \in \mathbb{B}_{E}, z \neq w} \omega\left(\left(1-|z|^{2}\right)^{\beta}\left(1-|w|^{2}\right)^{\alpha-\beta}\right) \\
& \quad \cdot \frac{|f(z)-f(w)|}{|z-w|} \leq C \epsilon,
\end{aligned}
$$

whenever $\delta<|z|<1$. Hence

$$
\lim _{|z| \rightarrow 1^{-}} \omega\left(\left(1-|z|^{2}\right)^{\alpha}\right) \Lambda_{f}(z)=0
$$

Necessity. For $t \in(0,1)$, let $f_{t}(z)=f(t z)$. By the proof of Theorem 9, we have

$$
\begin{gathered}
\omega\left(\left(1-|z|^{2}\right)^{\beta}\left(1-|w|^{2}\right)^{\alpha-\beta}\right) \\
\cdot \frac{\left|\left(f-f_{t}\right)(z)-\left(f-f_{t}\right)(w)\right|}{|z-w|} \leq C\left\|f-f_{t}\right\|_{\omega, \alpha}, \\
\omega\left(\left(1-|z|^{2}\right)^{\beta}\left(1-|w|^{2}\right)^{\alpha-\beta}\right) \frac{\left|f_{t}(z)-f_{t}(w)\right|}{|z-w|} \\
<\frac{\omega\left(\left(1-|z|^{2}\right)^{\beta}\left(1-|w|^{2}\right)^{\alpha-\beta}\right)}{\omega\left(\left(1-|t z|^{2}\right)^{\beta}\left(1-|t w|^{2}\right)^{\alpha-\beta}\right)} \\
\cdot \omega\left(\left(1-|t z|^{2}\right)^{\beta}\left(1-|t w|^{2}\right)^{\alpha-\beta}\right) \frac{|f(t z)-f(t w)|}{t|z-w|} \\
\leq \frac{C \omega\left(\left(1-|z|^{2}\right)^{\beta}\left(1-|w|^{2}\right)^{\alpha-\beta}\right)}{\omega\left(\left(1-|t z|^{2}\right)^{\beta}\left(1-|t w|^{2}\right)^{\alpha-\beta}\right)}\|f\|_{\omega, \alpha}
\end{gathered}
$$

for all $z, w \in \mathbb{B}_{E}$. By the triangle inequality, we have

$$
\begin{gathered}
\sup _{z, w \in \mathbb{B}_{E}, z \neq w} \omega\left(\left(1-|z|^{2}\right)^{\beta}\left(1-|w|^{2}\right)^{\alpha-\beta}\right) \\
. \frac{|f(z)-f(w)|}{|z-w|} \leq C\left\|f-f_{t}\right\|_{\omega, \alpha} \\
+\frac{C \omega\left(\left(1-|z|^{2}\right)^{\beta}\left(1-|w|^{2}\right)^{\alpha-\beta}\right)}{\omega\left(\left(1-|t z|^{2}\right)^{\beta}\left(1-|t w|^{2}\right)^{\alpha-\beta}\right)}\|f\|_{\omega, \alpha} .
\end{gathered}
$$

In the above inequality, first by letting $|z| \rightarrow 1^{-}$and then letting $t \rightarrow 1^{-}$, we obtain the desired result.

In the following, by adding the restriction $w \in E(z, r)$, we generalize [10, Theorems 1 and 2] to the following forms.

Theorem 11. Let $r \in(0,1), f \in \mathscr{P}\left(\mathbb{B}_{E}\right)$, and $0<\beta \leq \alpha$. Then $f \in \mathscr{B}_{\omega}^{\alpha}$ if and only if

$$
\begin{aligned}
K= & \sup _{w \in E(z, r), z \neq w} \omega\left(\left(1-|z|^{2}\right)^{\beta}\left(1-|w|^{2}\right)^{\alpha-\beta}\right) \\
& \cdot \frac{|f(z)-f(w)|}{|z-w|}<\infty .
\end{aligned}
$$

Proof. We only need to prove the necessity since the sufficiency easily follows from the proof of Theorem 9. Assume that $f \in \mathscr{B}_{\omega}^{\alpha}$. Then for any $w \in E(z, r), z \neq w$, we have

$$
\begin{aligned}
& |f(z)-f(w)|=\left|\int_{\overline{z w}} f_{\zeta}(\zeta) d \zeta+f_{\bar{\zeta}}(\zeta) d \bar{\zeta}\right| \\
& \quad \leq C|z-w| \int_{0}^{1} \frac{d s}{\omega\left(\left(1-|s z+(1-s) w|^{2}\right)^{\alpha}\right)} .
\end{aligned}
$$

Since, for $s \in[0,1]$,

$$
\begin{aligned}
1-|s z+(1-s) w|^{2} \geq & 1-|s z+(1-s) w| \\
\geq & s(1-|z|)+(1-s)(1-|w|) \\
\geq & s\left(\frac{1-|z|^{2}}{2}\right) \\
& +(1-s)\left(\frac{1-|w|^{2}}{2}\right) \\
\geq & \frac{1}{2}\left(1-|z|^{2}\right)^{s}\left(1-|w|^{2}\right)^{1-s},
\end{aligned}
$$

by Lemma 2, we obtain that

$$
\begin{aligned}
& \frac{|f(z)-f(w)|}{|z-w|} \\
& \leq C \int_{0}^{1} \frac{d s}{\omega\left(\left(1 / 2^{\alpha}\right)\left(1-|z|^{2}\right)^{\alpha s}\left(1-|w|^{2}\right)^{\alpha-\alpha s}\right)} \\
& \leq \frac{C}{\omega\left(\left(1-|z|^{2}\right)^{\alpha}\right)} \\
& \leq \frac{C}{\omega\left(\left(1-|z|^{2}\right)^{\beta}\left(1-|w|^{2}\right)^{\alpha-\beta}\right)} .
\end{aligned}
$$

Thus,

$$
\begin{gathered}
\sup _{w \in E(z, r), z \neq w} \omega\left(\left(1-|z|^{2}\right)^{\beta}\left(1-|w|^{2}\right)^{\alpha-\beta}\right) \\
. \frac{|f(z)-f(w)|}{|z-w|}<\infty .
\end{gathered}
$$

This completes the proof of Theorem 11 . 
Similarly, we can obtain the following.

Theorem 12. Let $r \in(0,1), f \in H\left(\mathbb{B}_{E}\right)$, and $0<\beta \leq \alpha$. Then $f \in \mathscr{B}_{\omega, 0}^{\alpha}$ if and only if

$$
\begin{gathered}
\lim _{|z| \rightarrow 1^{-}} \sup _{w \in E(z, r), z \neq w} \omega\left(\left(1-|z|^{2}\right)^{\beta}\left(1-|w|^{2}\right)^{\alpha-\beta}\right) \\
\cdot \frac{|f(z)-f(w)|}{|z-w|}=0 .
\end{gathered}
$$

\section{Conflicts of Interest}

The authors declare that they have no conflicts of interest.

\section{Acknowledgments}

The work was supported by the NNSF of China (nos. 10771121, 11301220, 11401387, and 11661052), the NSF of Zhejiang Province, China (no. LQ 14A010007), the NSF of Shandong Province, China (no. ZR2012AQ020), and the Fund of Doctoral Program Research of Shaoxing College of Art and Science (20135018).

\section{References}

[1] K. M. Dyakonov, "Equivalent norms on Lipschitz-type spaces of holomorphic functions," Acta Mathematica, vol. 178, no. 2, pp. 143-167, 1997.

[2] V. Lappalainen, "Liph-extension domains," Annales Academiae Scientiarum Fennicae. Series A I. Mathematica Dissertationes, no. 56, article 52, 1985.

[3] M. Pavlović, “On Dyakonov's paper "Equivalent norms on Lipschitz-type spaces of holomorphic functions", Acta MAThematica, vol. 183, no. 1, pp. 141-143, 1999.

[4] S. Chen, S. Ponnusamy, and A. Rasila, "On characterizations of Bloch-type, Hardy-type and Lipschitz-type spaces," Mathematische Zeitschrift, vol. 279, no. 1-2, pp. 163-183, 2015.

[5] K. M. Dyakonov, "Holomorphic functions and quasiconformal mappings with smooth moduli," Advances in Mathematics, vol. 187, no. 1, pp. 146-172, 2004.

[6] J. Qiao and X. Wang, "Lipschitz-type spaces of pluriharmonic mappings," Filomat, vol. 27, no. 4, pp. 693-702, 2013.

[7] O. Blasco, P. Galindo, and A. Miralles, "Bloch functions on the unit ball of an infinite dimensional Hilbert space," Journal of Functional Analysis, vol. 267, no. 4, pp. 1188-1204, 2014.

[8] K. Goebel and S. Reich, Uniform Convexity, Hyperbolic Geometry and Nonexpansive Mappings, vol. 83, Marcel Dekker, New York, NY, USA, 1984.

[9] G. Ren and C. Tu, "Bloch space in the unit ball of $\mathbb{C}^{n}$," Proceedings of the American Mathematical Society, vol. 133, no. 3, pp. 719-726, 2005.

[10] S. Li and H. Wulan, "Characterizations of $\alpha$-Bloch spaces on the unit ball," Journal of Mathematical Analysis and Applications, vol. 343, no. 1, pp. 58-63, 2008.

[11] R. Zhao, "A characterization of Bloch-type spaces on the unit ball of $\mathbb{C}^{n}$," Journal of Mathematical Analysis and Applications, vol. 330, no. 1, pp. 291-297, 2007.

[12] S. Chen and X. Wang, "On harmonic Bloch spaces in the unit ball of $\mathbb{C}^{n}$," Bulletin of the Australian Mathematical Society, vol. 84, no. 1, pp. 67-78, 2011.
[13] G. Ren and U. Kähler, "Weighted Lipschitz continuity and harmonic Bloch and BESov spaces in the real unit ball," Proceedings of the Edinburgh Mathematical Society. Series II, vol. 48, no. 3, pp. 743-755, 2005.

[14] R. Yoneda, "A characterization of the harmonic Bloch space and the harmonic BESov spaces by an oscillation," Proceedings of the Edinburgh Mathematical Society. Series II, vol. 45, no. 1, pp. 229239, 2002.

[15] K. Zhu, Spaces of Holomorphic Functions in the Unit Ball, Springer, New York, NY, USA, 2005.

[16] D. Kalaj and M. Vuorinen, "On harmonic functions and the Schwarz lemma," Proceedings of the American Mathematical Society, vol. 140, no. 1, pp. 161-165, 2012. 


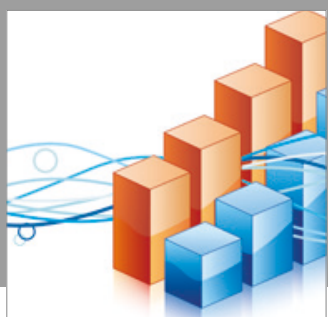

Advances in

Operations Research

vatersals

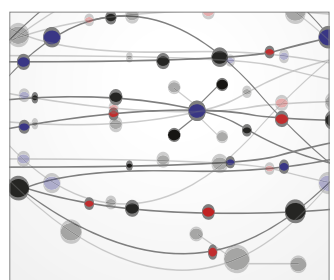

\section{The Scientific} World Journal
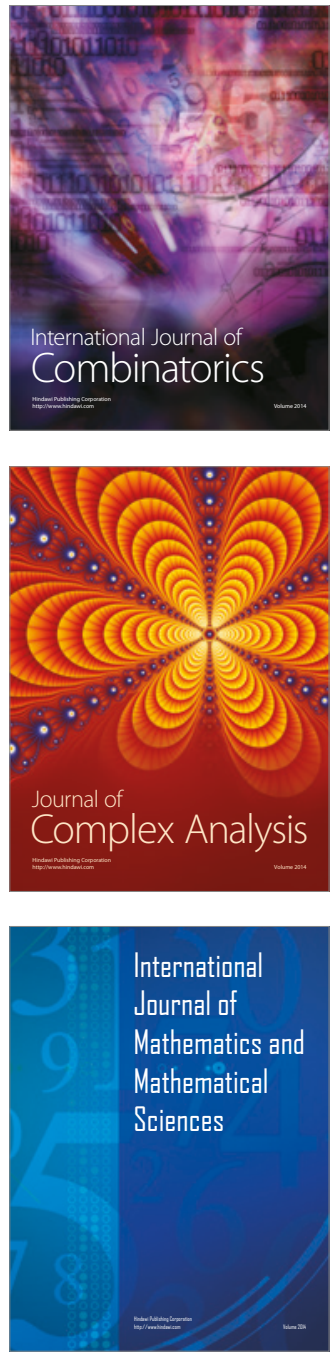
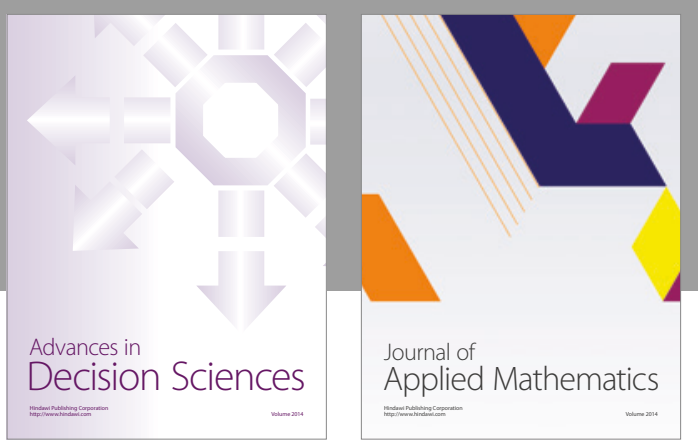

Algebra

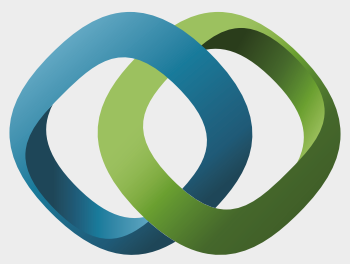

\section{Hindawi}

Submit your manuscripts at

https://www.hindawi.com
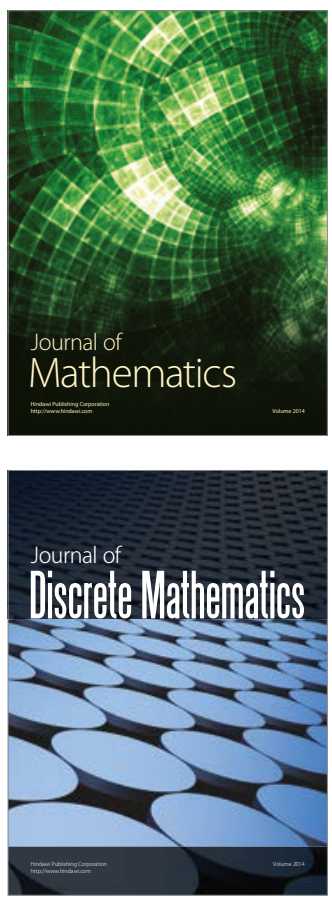

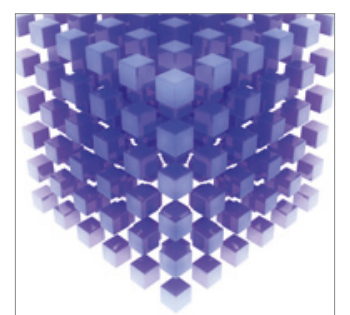

Mathematical Problems in Engineering
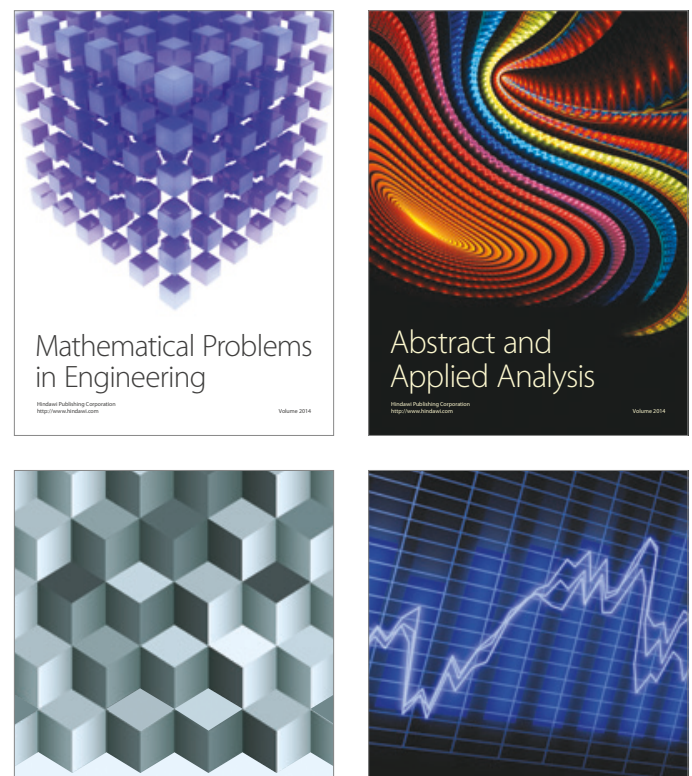

Journal of

Function Spaces

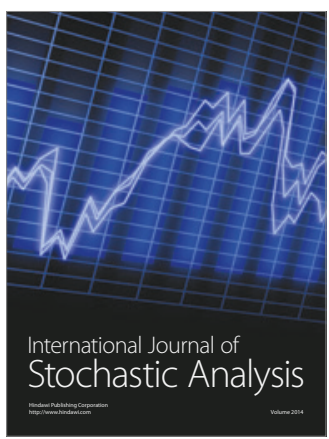

Probability and Statistics
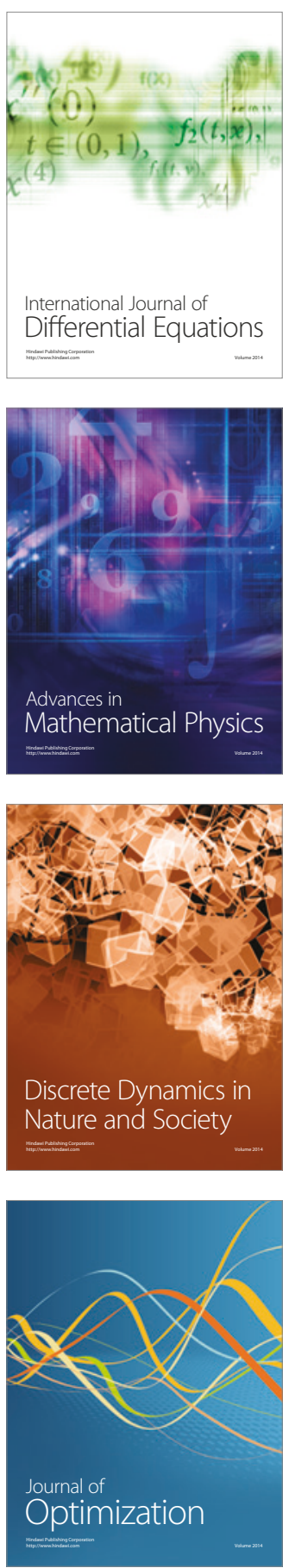